\title{
EXPERIMENTAL AND NUMERICAL ANALYSIS OF MR DAMPERS
}

\author{
Manuel T. Braz-César ${ }^{1}$, Rui C. Barros ${ }^{2}$ \\ ${ }^{1}$ Polytechnic Institute of Bragança \\ Campus de Santa Aplónia - Apt. 134 - 5301-857 Bragança - Portugal \\ e-mail: brazcesar@ipb.pt \\ ${ }^{2}$ Faculty of Engineering of the University of Porto \\ Rua Dr. Roberto Frias, s/n 4200-465 Porto - Portugal \\ rcb@fe.up.pt
}

Keywords: MR damper, Bouc-Wen model.

Abstract. This paper provides a brief description of MR fluid technology and MR fluid based devices. Analytical and experimental investigations about MR dampers covering their rheological properties and hysteretic behavior will be carried out. In the first section an overview of the basic properties of the MR fluids and the fluid behavior under different flow regimes are presented. Then, a selection of numerical models to simulate MR dampers behavior will be presented based on the available literature. To obtain and analyze the hysteretic behavior of a MR damper, a commercial device was experimentally tested under several input excitations. Based on the experimental results an identification procedure was carried out to determine the necessary parameters to develop a numerical model. Finally, results from experimental investigations and numerical analyses are summarized and compared. 


\section{INTRODUCTION}

The so-called "smart materials" has received the attention of researchers and engineers due to their capability to create smart devices that can be easily controlled by a small external perturbation like temperature or a magnetic field. Some of the most promising smart devices are based on fluids with controllable properties like Electrorheological (ER) and Magnetorheological (MR) fluids. The performance of MR fluids is less sensitive to temperature because the magnetic polarization mechanism remains unchanged over the operating temperature range and MR fluids behaviour is not affected by impurities, which means that is insensitive to contamination, while ER fluids are highly sensitive to moisture or impurities as result of manufacture and usage process.

MR fluids are non-Newtonian and rheologically stable suspensions with a shear yield strength, which can be controlled by a magnetic field. These fluids react promptly to the application of an external magnetic field (in a few milliseconds) exhibiting a reversible and adjustable transition from a free-flowing state to a semi-solid state. Due to this property these materials exhibit a significant change in their rheological behavior (viscosity and plasticity).

The expression "apparent viscosity" is used because the carrier fluid viscosity does not change as the magnetic field intensity $\mathrm{H}$ is modified. The rheological behavior of MR fluids depends on the magnetic field strength $\mathrm{H}$, however it is possible to define a pre and post yield areas as shown in Figure 1a. In the pre-yield region the MR fluid exhibits visco-elastic behavior and in the post-yield region it behaves like a viscous Newtonian fluid.
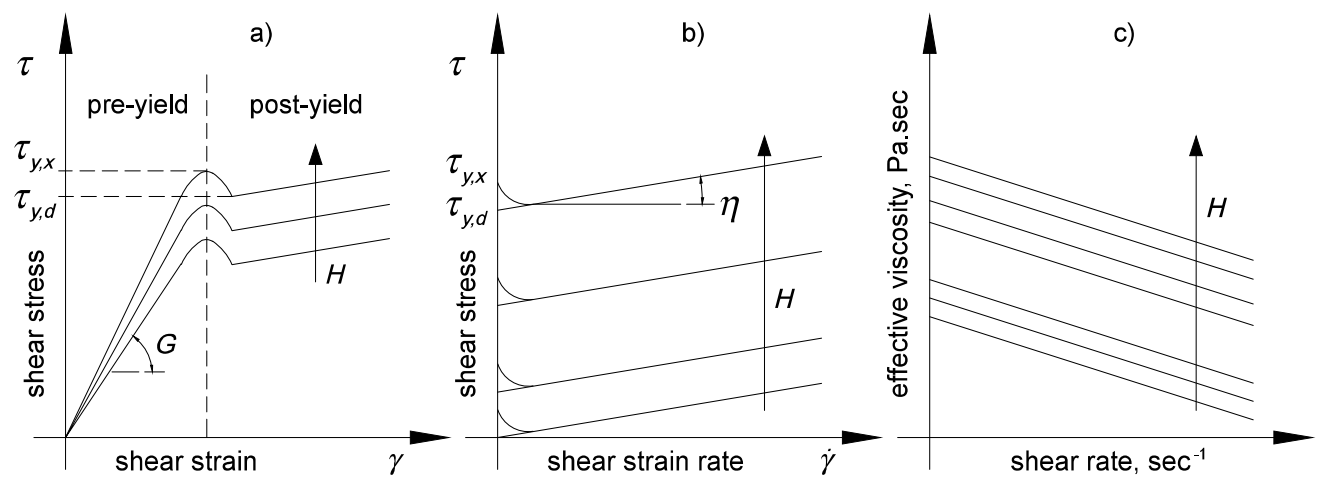

Figure 1: Constitutive behavior of a MR fluid: a) pre-yield and post-yield regions, b) non-Newtonian post-yield behavior and c) apparent viscosity.

In can be stated that the transition from pre-yield to the post-yield region occurs when the stress is greater than the yield shear stress. In this transition zone it is possible to identify the dynamic shear yield stress $\tau_{y, d}$ defined as the point of the zero-rate and the static shear yield stress $\tau_{y, s}$ defined as the shear stress necessary to initiate MR fluid flow $[1,2]$.

\section{EXPERIMENTAL RESPONSE}

The device employed in the testing, identification and modeling of the non-linear hysteretic response is the RD-1005-3 commercial prototype developed by the LORD Corporation.

An extensive experimental research was developed to evaluate and obtain the MR damper response data necessary to proceed with the identification procedure [3, 4]. The experimental tests were performed using a MTS universal testing machine load frame with a MTS load unit controller. 
The LORD MR damper are usually commanded through a voltage-to-current controller unit designated as Wonder Box Device Controller and sold as a complementary product for MR devices. The device provides a closed-loop current control to compensate for changing electrical loads up to the limits of the power supply and can be used to investigate the control possibilities of the LORD MR technology since it can be operated as an interface device for PLC or computer control of the MR fluid devices. This controller was connected to a voltage power supply unit in order to feed the MR damper with a constant voltage converted to current supply through the Wonder Box.

The RD-1005-3 MR damper has a conventional cylindrical body configuration filled with $50 \mathrm{ml}$ of MR fluid and comprising the piston, the magnetic circuit with a coil resistance of 5 $\Omega$ and the accumulator. The enclosing cylinder is $41.4 \mathrm{~mm}$ in diameter and the damper is 208 $\mathrm{mm}$ long in its extended position with $\pm 2.5 \mathrm{~cm}$ stroke. The device can operate within a current range from $0.0 \mathrm{~A}$ up to $2.0 \mathrm{~A}$ with a recommended input value of $1.0 \mathrm{~A}$ for continuous operation and can deliver a peak force of $2224 \mathrm{~N}$ at a velocity of $51 \mathrm{~mm} / \mathrm{s}$ with a continuous operating current level of $1.0 \mathrm{~A}$. The MR damper can reach at least $90 \%$ of maximum level during a $0.0 \mathrm{amp}$ to $1.0 \mathrm{amp}$ step input in less than 25 milliseconds.

A series of sinusoidal displacement excitation tests were performed to measure the response under different loading conditions in order to obtain the hysteretic response of the MR damper. The MR damper was mounted into the MTS hydraulic actuation system and was then excited with a sinusoidal displacement. An extensive parametric study was carried out for several arrangements of amplitudes, frequencies and input current; these scenarios were studied in order to obtain the required experimental data to conveniently characterize the damper response $[5,6]$.

The selected parameters to develop the test program in order to determine the steady state characteristics of the damper for sinusoidal inputs comprise the set of frequencies, amplitudes and current supplies specified in Table 1. To refine the experimental response of the MR damper, the applied current ranges from $0.0 \mathrm{~A}$ to a maximum of $1.0 \mathrm{~A}$ in increments of $0.25 \mathrm{~A}$. The input current range was refined from $0.0 \mathrm{~A}$ to $0.25 \mathrm{~A}$ to conveniently characterize the current dependent MR damper response at low current input.

\begin{tabular}{|c|c|}
\hline Parameter & Values \\
\hline Frequencies $(\mathrm{Hz})$ & $(0.50,1.00,1.50,2.00)$ \\
\hline Amplitudes $(\mathrm{mm})$ & $(2.0,4.0,6.0,8.0,10.0)$ \\
\hline Current supplies $(\mathrm{A})$ & $(0.00,0.10,0.20,0.25,0.50,0.75,1.00)$ \\
\hline
\end{tabular}

Table 1: Sinusoidal signal - Lord RD-1005-3 MR damper experimental analysis

The testing procedure was carried out with a fixed frequency and amplitude sinusoidal displacement for a specific current supply, repeating this process for every parameter combination. The experimental data of the parametric study for MR dampers are typically grouped according to the variability of the different parameters sets as frequency-dependent tests and amplitude-dependent tests.

The sinusoidal shaft displacement and the resulting force are measured though the MTS internal LVDT and a force transducer (load cell) with a sampling rate of $256 \mathrm{~Hz}$ and logged for data analysis. The displacement and force transducers were calibrated previously to the experimental procedure to reduce measuring imprecisions. Based on the results it is possible to generate the force-displacement (f-d) and force-velocity (f-v) hysteresis cycles of the damper and therefore understand and investigate the device rheological behavior. 
Figures 2 illustrate some of the experimental results obtained with the testing procedure. The plots were developed from the data records selecting an experimental response cycle for a steady state excitation.
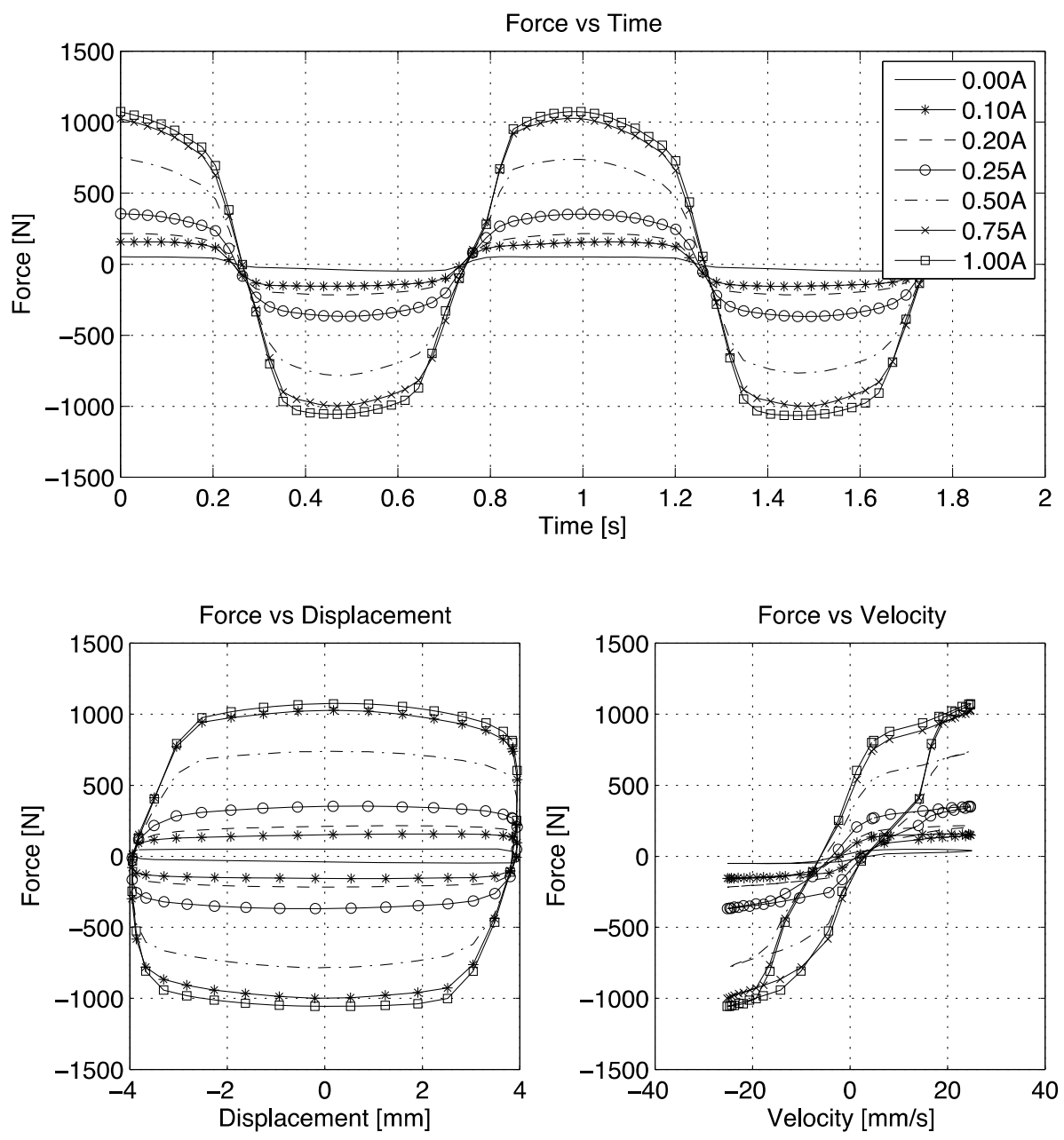

Figure 2: RD-1005-3 MR damper - Measured damping forces under a $1.00 \mathrm{~Hz}$ sinusoidal excitation with amplitude of $4.00 \mathrm{~mm}$ and variable input current.

\section{NUMERICAL MODELS}

MR dampers are semi-active devices whose damping characteristics can be modified in real time due to the capability to adjust the resistance to flow of a MR fluid within the damper through the application of a magnetic field. The particular properties of the MR fluid allow variations in the damping force that can be controlled by varying an applied current. Thus, the hysteretic behaviour of a MR damper is current dependent but also function of the amplitude of the excitation.

To predict the behaviour of MR dampers under certain magnetic fields or excitations, it is necessary to model the device with an appropriate approach. Hence a previous numerical simulation is needed to verify if the proposed properties are suitable for the system in which it is intended to apply the MR damper. Several numerical models are available to predict the response of MR dampers [7-11]. Table 2 presents a brief description of the available models for MR dampers. 


\begin{tabular}{|c|c|}
\hline Modelling technique & MR damper Models \\
\hline Bingham models & $\begin{array}{l}\text { - Original Bingham model } \\
\text { - Modified Bingham model } \\
\text { - Gamota and Filisko model } \\
\text { - Updated Bingham model by Occhiuzzi et al. } \\
\text { - Three-element model by Powell }\end{array}$ \\
\hline Bi-viscous models & $\begin{array}{l}\text { - Nonlinear bi-viscous model } \\
\text { - Nonlinear hysteretic bi-viscous model } \\
\text { - Nonlinear hysteretic arctangent model } \\
\text { - Lumped parameter bi-viscous model }\end{array}$ \\
\hline Visco-elastic-plastic models & $\begin{array}{l}\text { - General visco-elastic-plastic models } \\
\text { - Visco-elastic-plastic model by Li et al }\end{array}$ \\
\hline Stiffness-viscosity-elasto-slide model & - Stiffness-viscosity-elasto-slide (SVES) model \\
\hline Hydro-mechanical model & - Hydro-mechanical model \\
\hline Maxwell models & $\begin{array}{l}\text { - BingMax model by Makris et al. } \\
\text { - Maxwell Nonlinear Slider model }\end{array}$ \\
\hline Bouc-Wen models & $\begin{array}{l}\text { - Simple Bouc-Wen model } \\
\text { - Modified Bouc-Wen model } \\
\text { - Bouc-Wen model for shear mode dampers } \\
\text { - Bouc-Wen model for large-scale dampers } \\
\text { - Current dependent Bouc-Wen model } \\
\text { - Current-frequency-amplitude dependent Bouc-Wen } \\
\text { - Non-symmetrical Bouc-Wen model }\end{array}$ \\
\hline Dahl models & $\begin{array}{l}\text { - Modified Dahl model } \\
\text { - Viscous Dahl model }\end{array}$ \\
\hline LuGre models & $\begin{array}{l}\text { - Modified LuGre model by Jimenez and Alvarez } \\
\text { - Modified LuGre model by Sakai et al }\end{array}$ \\
\hline Hyperbolic tangent models & - Hyperbolic tangent model by Kwok et al \\
\hline Sigmoid models & - Sigmoid model by Wang et al and Ma et al \\
\hline Equivalent models & - Equivalent model by Oh and Onoda \\
\hline Phase transition models & - Phase transition model \\
\hline
\end{tabular}

Table 2 - MR dampers Models classification

The main problem regarding the modeling of MR dampers is related with the significant non-linear response that these devices exhibit when subjected to an input excitation. Moreover, the hysteretic behavior observed is function of the amplitude and frequency of excitation but (due to their rheological nature and due to the semi-active controllable response) it is also current dependent, which considerably increases the difficulty level when a numerical model is developed.

To use the MR damper as a controllable semi-active device into a control system it is essential that the selected numerical model can capture its non-linear behavior in order to develop a feasible semi-active controller [3-6].

Since many mathematical models have been developed to describe such behavior and to take advantage of the MR properties of these devices in vibration control related problems, it is necessary to select and implement high-accuracy models capable to capture their non-linear hysteretic response. Among the modeling techniques, parametric models appear to be an easy and reliable approach to obtain a mathematical model of the physical MR damper.

Figure 3 illustrates the response of well-known Bingham, Bouc-Wen and Modified BoucWen models [12-14], which are some of the most common models obtained by a deterministic approach. 
The Bingham mechanical model consists of a Coulomb friction element placed in parallel with a viscous damper and comprises the identification of three parameters (Figure 3): the frictional force $f_{c}$, the viscous damping parameter $c_{0}$ and the force due to the presence of the accumulator $f_{0}$.

The simple Bouc-Wen model presents a highly effective model for hysteretic systems and has become one of the most common approaches to describe the hysteretic behavior of MR dampers. The force generated by the MR damper can be expressed by the combination of three elements (Figure 3): a dashpot defined by the damping coefficient $c_{0}$, a linear spring defined by the parameter $k_{0}$ and a Bouc-Wen component defined by the Bouc-Wen parameter $\alpha$ that represent the stiffness for the damping force component (yield stress of the MR fluid) associated with the evolutionary variable $z$ that accounts for the history dependence of the response.

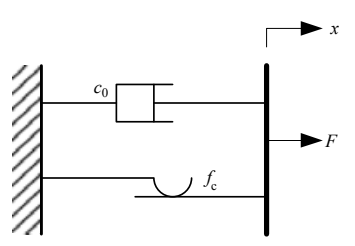

a) $\quad F(t)=c_{0} \dot{x}+f_{c} \operatorname{sgn}(\dot{x})+f_{0}$
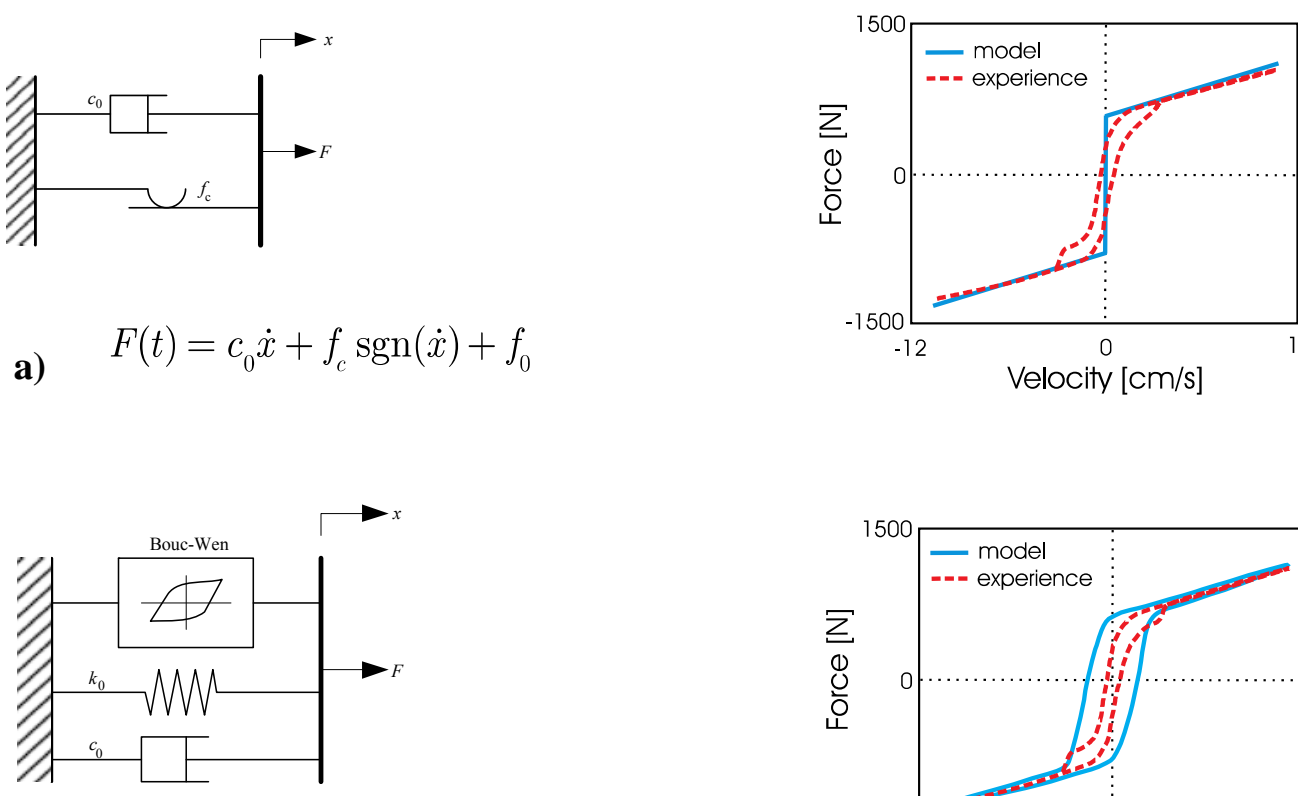

b) $\quad F(t)=c_{0} \dot{x}+k_{0}\left(x-x_{0}\right)+\alpha z$
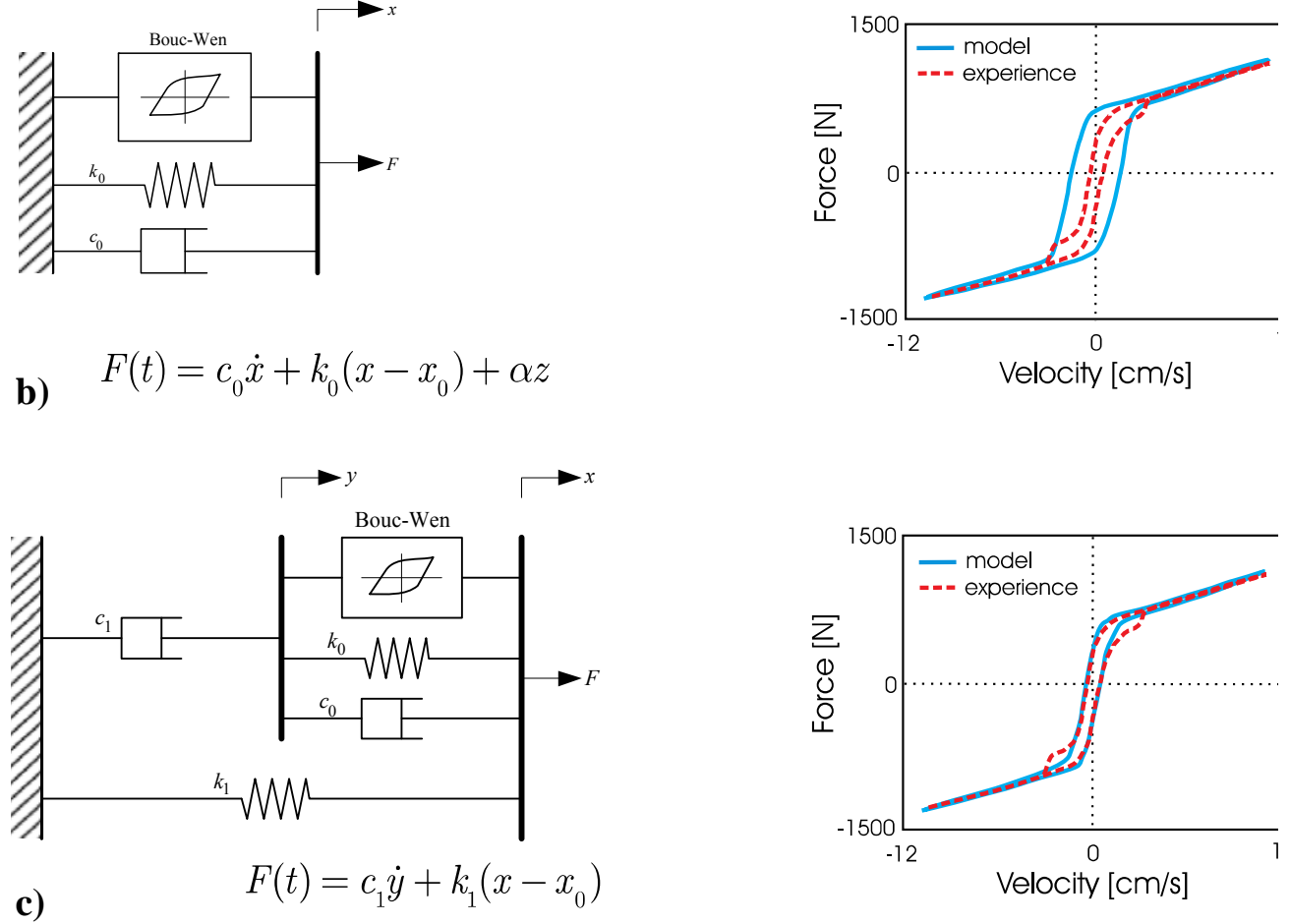

Figure 3: MR-fluid and MR-dampers models [12, 13]

a) Bingham model, b) Bouc-Wen model, c) Modified Bouc-Wen model 
The non-linear hysteretic behavior of the device is controlled by the Bouc-Wen component that is related with the restoring force of a non-linear hysteretic system as proposed by [15]. According to the Bouc-Wen formulation, the restoring force of the nonlinear hysteretic system $Q(x, \dot{x})$ can be decomposed into two parts as

$$
Q(x, \dot{x})=g(x, \dot{x})+z(x)
$$

where $g(x, \dot{x})$ is a non-hysteretic component expressed by the displacement $x$ and velocity $\dot{x}$, while $z(x)$ is a hysteretic component defined by the displacement and the evolutionary variable $\mathrm{z}$ given by

$$
\begin{array}{ll}
\dot{z}=-\beta|\dot{x}| z^{n}-\gamma \dot{x}|z|^{n}+A \dot{x}, & n=1,3,5, \ldots \\
\dot{z}=-\beta|\dot{x}| z^{n-1}-\gamma \dot{x} z^{n}+A \dot{x}, & n=2,4,6, \ldots
\end{array}
$$

These equations are implemented in the simple Bouc-Wen model with an equivalent formulation in which the evolutionary variable is written as

$$
\dot{z}(t)=-\beta|\dot{x}(t)| z(t)|z(t)|^{n-1}-\gamma \dot{x}(t)|z(t)|^{n}+A \dot{x}(t)
$$

The evolutionary variable equation depends on four parameters $A, \beta, \gamma$, and $n$ that represent the linearity in the unloading region and the smoothness of the transition from the pre-yield to post-yield regions.

Parameters $A, \beta$ and $\gamma$ describe the shape and size of the hysteresis loop and the parameter $n$ controls the smoothness of the transition from elastic to plastic response. To avoid numerical errors when $0<n<1$, the evolutionary variable equation can be rewritten in the following form

$$
\dot{z}(t)=\dot{x}(t)\left\{A-[\gamma+\beta \operatorname{sgn}(\dot{x}(t) \operatorname{sgn}(z(t)))]|z(t)|^{n}\right\}
$$

that can be derived as

$$
\frac{d z}{d x}=A-[\gamma+\beta \operatorname{sgn}(\dot{x}(t) \operatorname{sgn}(z(t)))]|z(t)|^{n}
$$

and the ultimate value of $z$ can be computed by setting $d z / d x=0$ and is given by

$$
z_{\max }=\left[\frac{A}{\beta+\gamma}\right]^{\frac{1}{n}}
$$

It can be stated that setting the non-hysteretic component $g(x, \dot{x})=0$ the restoring force will become $Q(x, \dot{x})=z(x)$. Then, if $z=0, d Q / d x=d z / d x=A$, and hysteretic curves for different excitation and response levels will have the same slope when $Q=Q(z)$ and the loading and unloading path are identical [15]. Thus, the non-hysteretic term must be used to overcome inconsistencies between the numerical model and the experimental results. The force generated by the MR damper can be expressed by the four parameters $(A, \beta, \gamma$, and $n)$ and a combination of the three parameters $c_{0}, k_{0}$ and $\alpha$ related with the physical components of the model.

The variable $x_{0}$ that appears in the Bouc-Wen model represent the initial displacement of the linear spring and is related with the force offset during the experimental tests. The evolutionary variable equation contains four parameters that have a strong effect on the hysteresis response curve. 
A comprehensive analysis of the influence of the Bouc-Wen parameters on the hysteretic behavior of this model was addressed in some previous research programs [16-18]. According with the Bouc-Wen formulation, the shape of the hysteretic loop is defined by parameters $A, \beta$, $\gamma$ and $n$. Parameters $A, \beta$ and $n$ are positive real numbers while $\gamma$ can be a positive or negative real number. The following study will be carried out for a harmonic periodic excitation $x=x_{0}$ $\sin (w(t))$, where $w=1 \mathrm{rad} / \mathrm{s}$ is the frequency and $x_{0}$ the amplitude of excitation.

The parameter $A$ defines the scale and amplitude of the hysteresis loop and also controls the slope of the hysteresis loop at $z=0$ (the stiffness characteristic) as shown in Figure 4. In this plot, the parameters $\beta$ and $\gamma$ were kept constant $(\beta=0.5$ and $\gamma=-0.4)$ and parametric variation of $A$ for a fixed value of $n=1.0$ was carried out. Increasing the parameter A will generate a larger hysteresis loop that implies an increase in the energy dissipation.

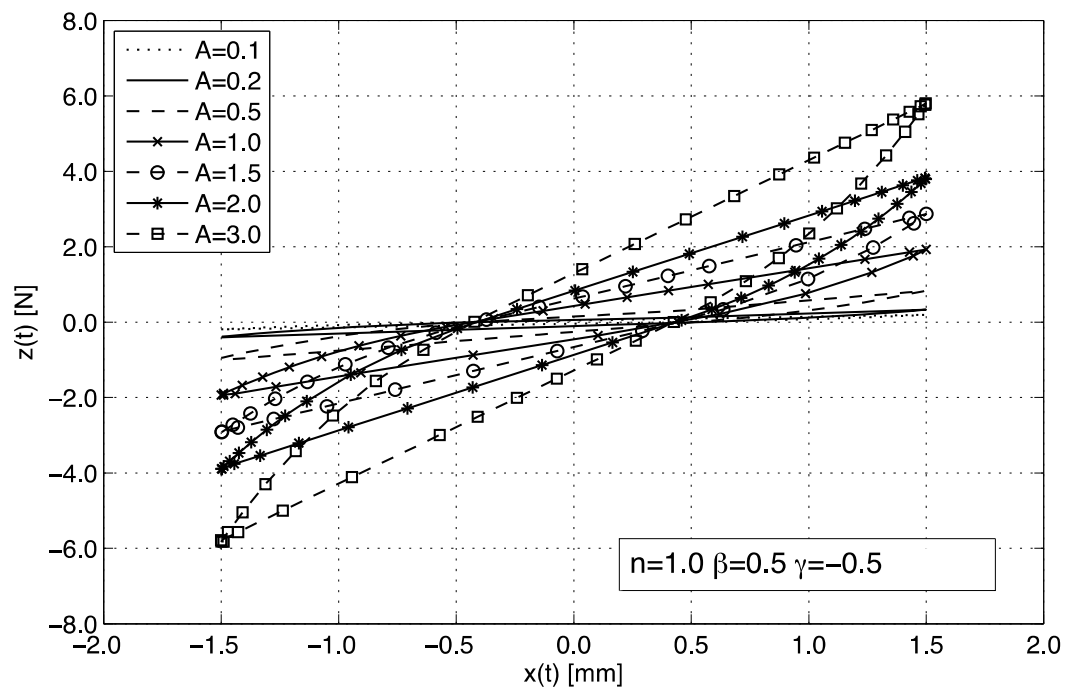

Figure 4: Influence of parameter A on the hysteretic behavior.

The parameter $n$ is related with the smoothness of the transition from the linear to the nonlinear region. The influence of this parameter in the hysteresis loop is shown in Figure 5.

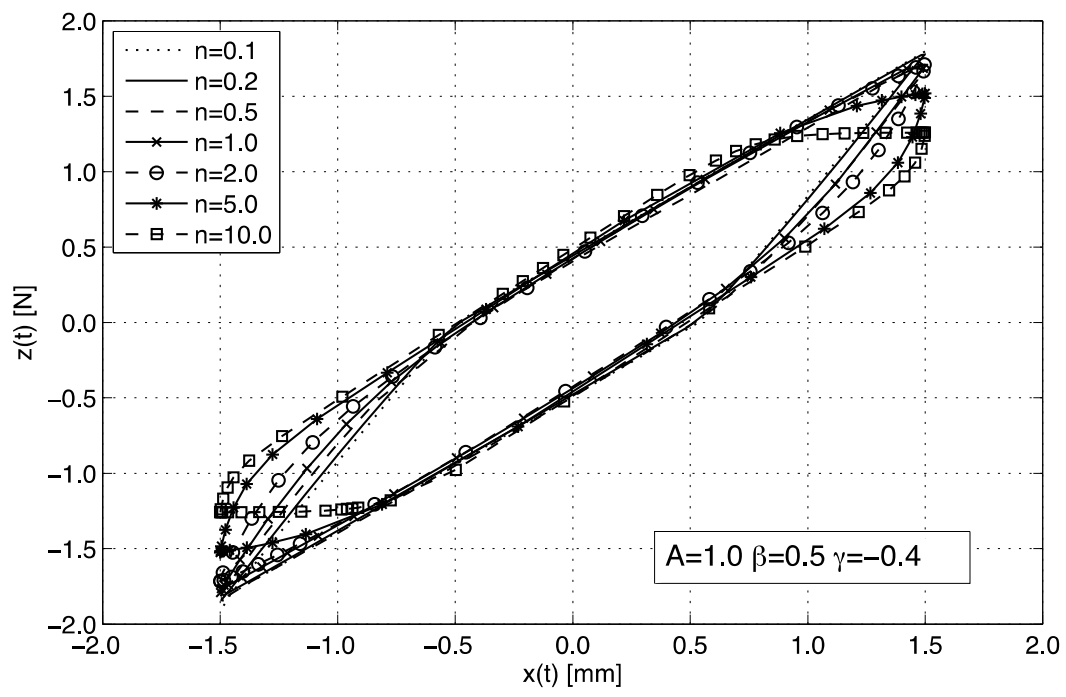

Figure 5: Influence of parameter $n$ on the hysteretic behavior. 
In this case, parameters $\beta, \gamma$ and $A$ are constant $(\beta=0.5, \gamma=-0.4$ and $A=1.0)$ while the parameter $n$ is changed. The parametric studies show that this power parameter $n$ has a significant effect on the hysteresis loop when $1<n<2$ and practically no effect when $n>2$.

The parameters $\beta$ and $\gamma$ are related with the shape of the hysteresis curve and different combinations of these parameters will describe several hysteretic loops with variations in the stiffness characteristics. The dependence of the hysteretic loops on the parameter $\beta$ is shown in Figure 6 for $A=1, \gamma=0.9$ and $n=1$. Essentially, the parameter $\beta$ controls the variation in the stiffness when the sign of the velocity variable changes. Hence, the response exhibits a linear hysteretic behavior when $\beta=0$, a hardening hysteretic behavior when $\beta<0$ and a softening hysteretic behavior when $\beta>0$.

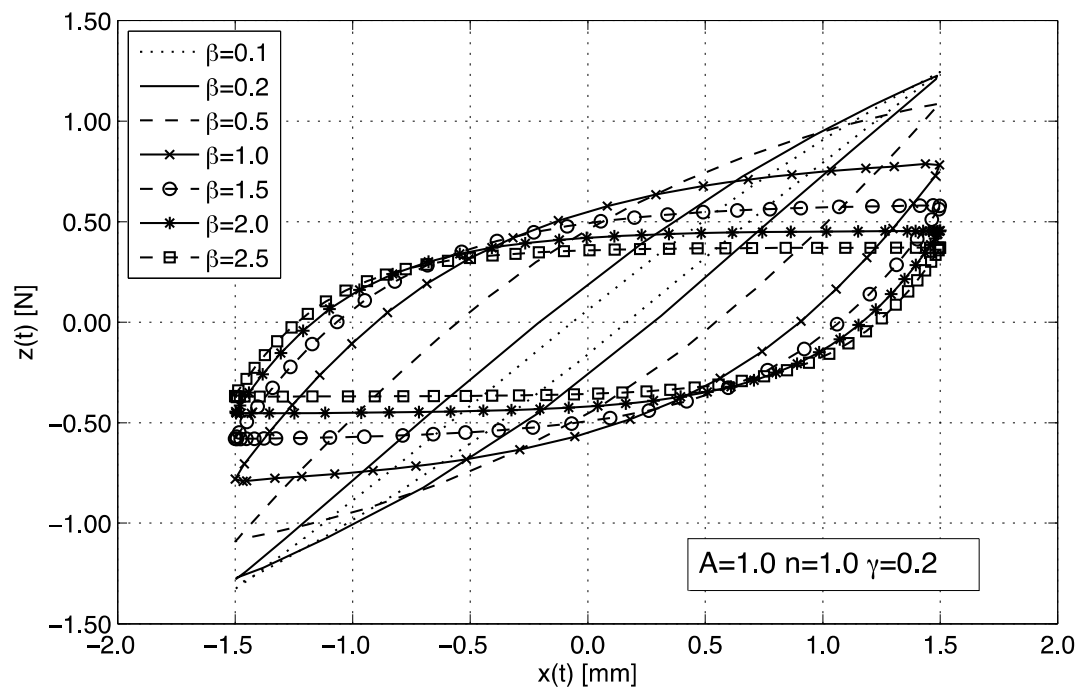

Figure 6: Influence of parameters $\beta$ on force-displacement curves.

The parameter $\gamma$ is related with the area and flatness of the hysteresis loop as displayed in Figure 7. Increasing the value of $\gamma$ will produce a decrease in the loop inclination producing a flat hysteretic curve with a near horizontal branch.

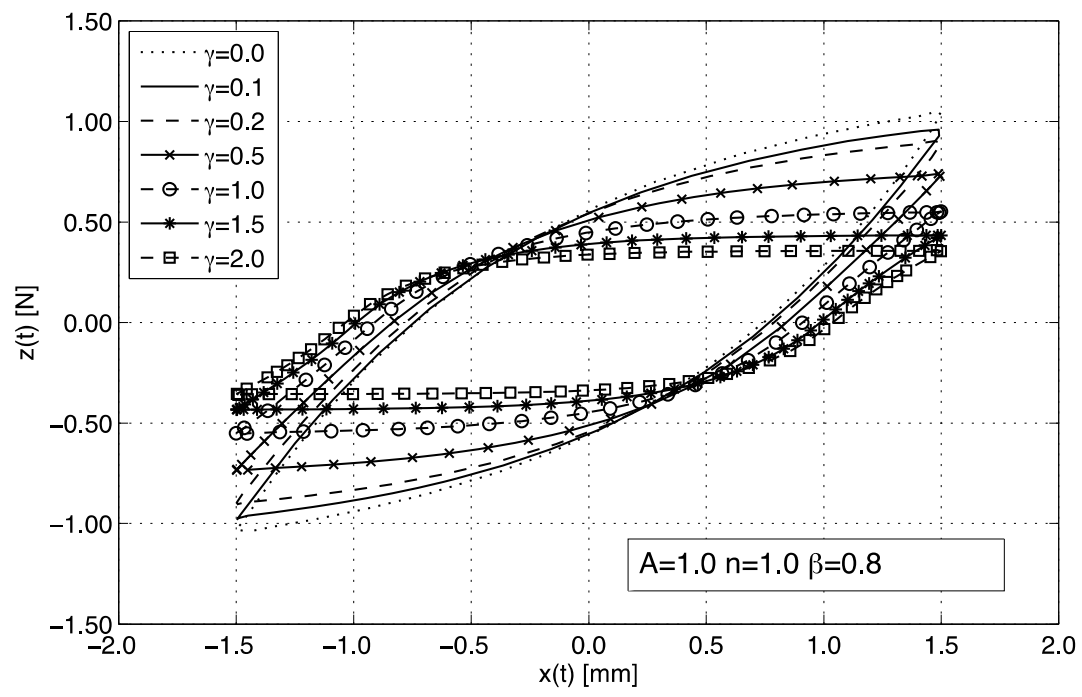

Figure 7: Influence of parameter $\gamma$ on the hysteretic behavior. 
Also, when the parameter is bellow a certain value defined as $\gamma_{0}$ the area will increase. However, when $\gamma$ is larger than $\gamma_{0}$, the hysteretic loop becomes almost horizontal and the area slightly decreases, i.e., the energy dissipated per cycle decreases. The parameters $\beta$ and $\gamma$ are related with the shape of the hysteresis curve and different combinations of these parameters will describe several hysteretic loops.

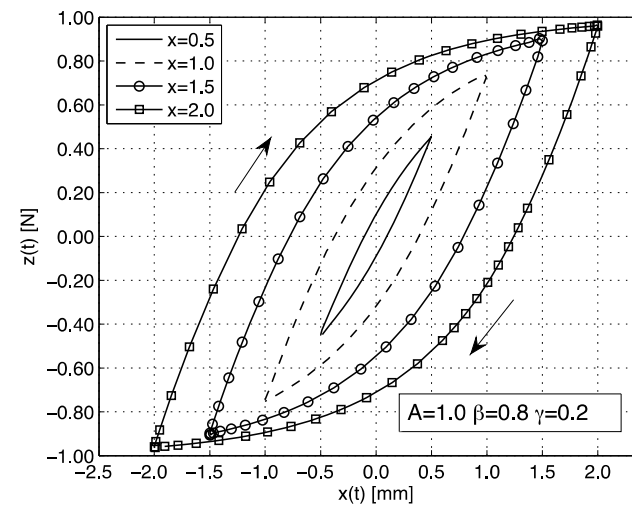

a) $(\beta+\gamma)>0$ and $(\beta-\gamma)>0$

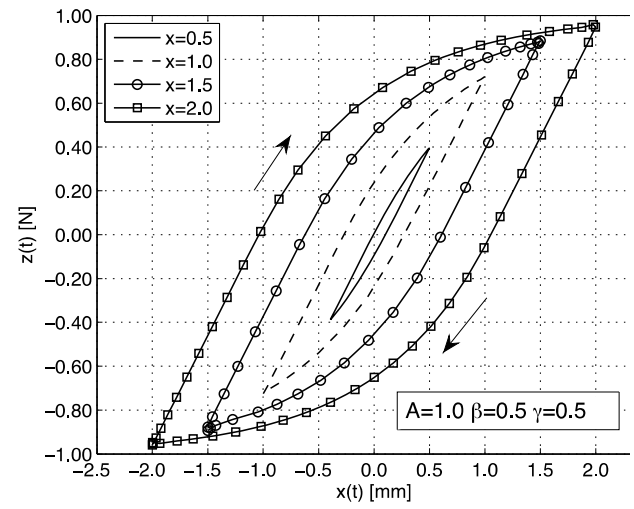

c) $(\beta+\gamma)>0$ and $(\beta-\gamma)=0$

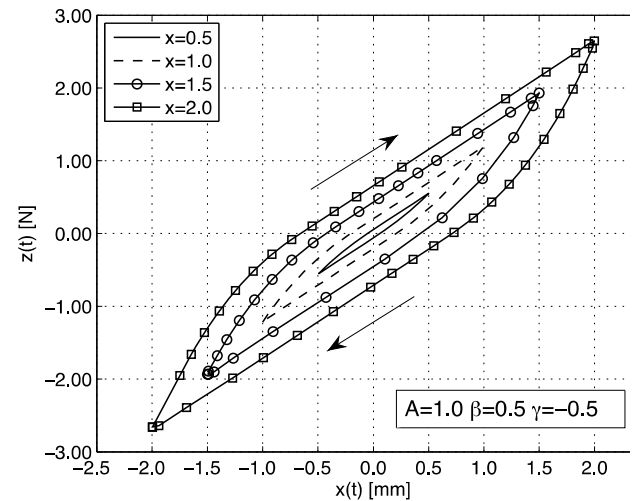

e) $(\beta+\gamma)=0$ and $(\beta-\gamma)>0$

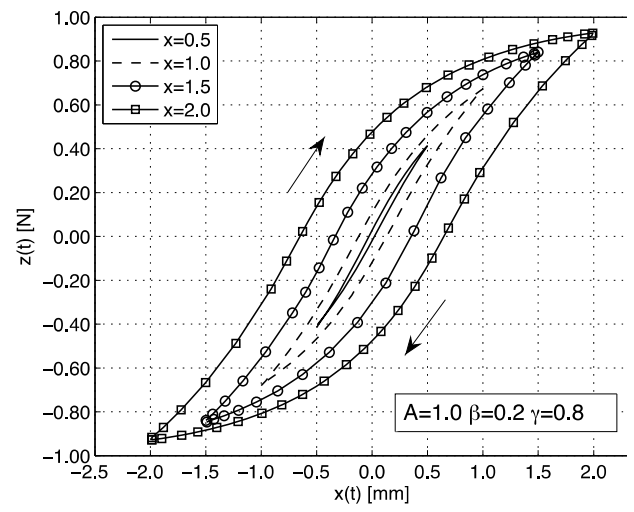

b) $(\beta+\gamma)>0$ and $(\beta-\gamma)<0$

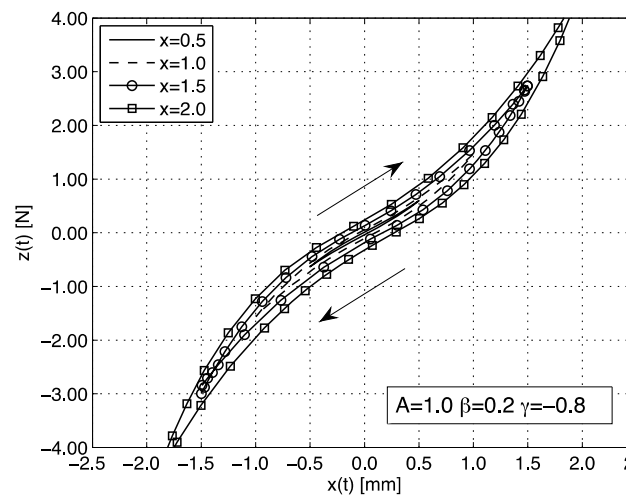

d) $(\beta+\gamma)<0$ and $(\beta-\gamma)>0$

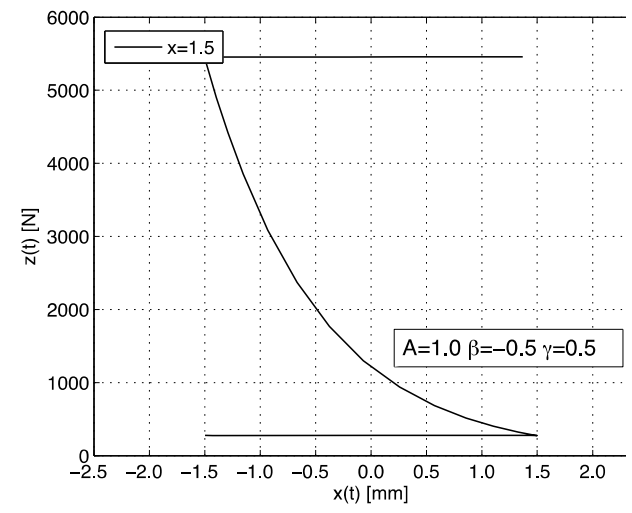

f) Other $(\beta+\gamma)=0$ and $(\beta-\gamma)<0$

Figure 8: Hysteresis loops for several combinations of $\beta$ and $\gamma$ 
Therefore, is important to study the relationship between these parameters to understand the influence of each combination in the hysteretic shape. The influence of the combination of these parameters in the hysteretic behavior can be studied investigating the slope $d z / d x$ of the hysteresis curve under loading and unloading.

When the loop is loading, the model show a softening non-linear behavior that occurs due to the fact that the slope of the hysteresis curve for $\mathrm{z}>0$ decreases with the increase of $z$ when $(\beta+\gamma)>0$ displaying a softening effect. The model exhibits hardening non-linearity produced by the increase in the slope of the hysteresis curve for $z>0$ with the increase of $\mathrm{z}$ when $(\beta+\gamma)<0$. Finally, the slope during loading for $z>0$ is kept constant and the system behaves quasi-linearly when $(\beta+\gamma)=0$. On the other hand, when the loop is unloading, the slope of the hysteresis curve for $\mathrm{z}>0$ decreases with the decrease of $\mathrm{z}$ when $(\beta-\gamma)>0$, increases with the decrease of $\mathrm{z}$ when $(\beta-\gamma)<0$ and is kept constant when $(\beta-\gamma)=0$ [6]. Eight possible combinations of $\beta$ and $\gamma$ can be used, however, only five of these combinations define hysteresis curves (or cases) that have physical meaning.

A parametric study for each case and the corresponding hysteresis loops are displayed in Figure $8[5,6]$. The parameters $A$ and $n$ were both kept constant at 1.0 and the arrow indicates the loading to unloading direction. In the first three cases is visible a softening non-linear behavior during loading as result of a decrease of the loop slope produced by an increase of $z$ (for positive values of $z$ ) when $(\beta+\gamma)>0$. In the fourth case the system reveals a hardening nonlinearity during loading as result of an increase of the loop slope produced by an increase of $z$ (with $z>0$ ) when $(\beta+\gamma)<0$. In the fifth and final case with physical meaning, the slope during loading for $z>0$ remain constant and the system presents a quasi-linear behavior when $(\beta+\gamma)=0$.

As expected, when the curve is in the unloading path, the slope for $z>0$ decreases with the decrease of $\mathrm{z}$ when $(\beta-\gamma)>0$, increases with the decrease of $\mathrm{z}$ when $(\beta-\gamma)<0$ and remain constant when $(\beta-\gamma)=0$. The hysteretic curves in Figures $7 \mathrm{a}$ and $7 \mathrm{c}$ have a similar shape and amplitude, which suggest that different combinations of $\beta$ and $\gamma$ can produce the same hysteretic response. Thus the use of the two parameters can be redundant and the combination of $\beta$ and $\gamma$ can be replaced by a single parameter $\beta$. Figure 9 shows the hysteretic loops for two combinations of $\beta$ and $\gamma(\beta=0.8, \gamma=0.2$ and $\beta=\gamma=0.5)$ to demonstrate that different combinations of these parameters can generate the same hysteretic behavior.

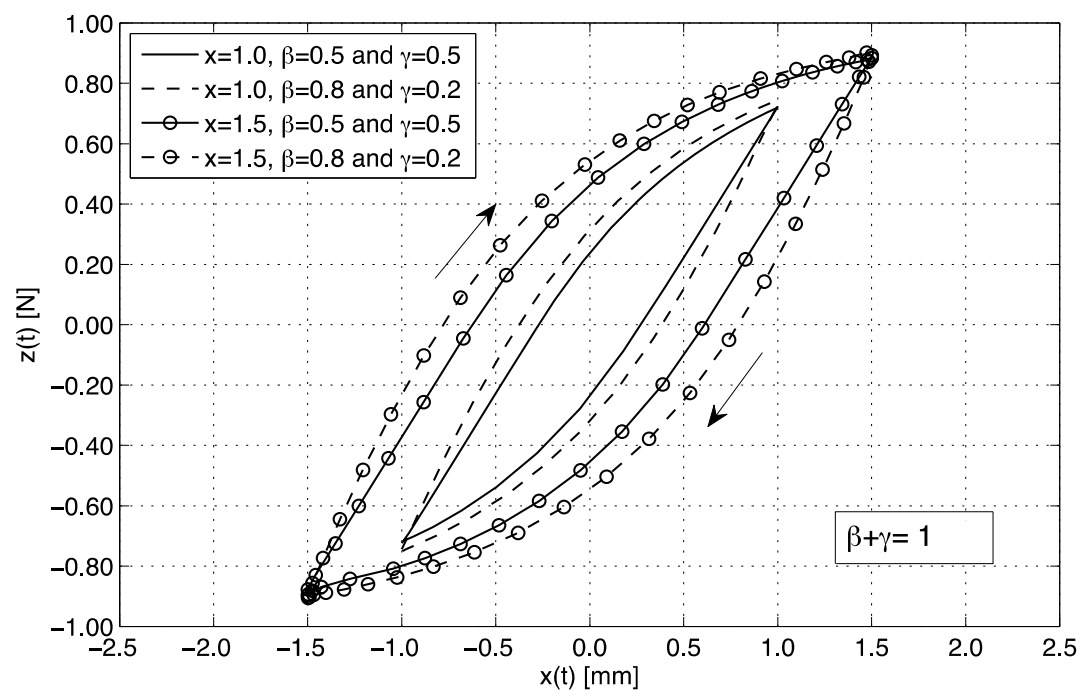

Figure 9: Hysteresis loops for two combinations of $\beta$ and $\gamma$ Comb1 $(\beta=0.8, \gamma=0.2)$ and Comb2 $(\beta=0.5, \gamma=0.5)$. 
In these combinations $(\beta+\gamma)=1$ and although a slight different in the hysteresis amplitude is present due to a larger value of $\beta$ in the first combination, the hysteretic response in both cases is almost identical and adjusting the value of each parameter it is possible to obtain equivalent curves. Therefore it is possible to justify that $\beta+\gamma$ is constant.

The relevance of the softening or hardening effect is determined by the combination of these parameters. Observing the third hysteretic curve, the system exhibits softening hysteretic behavior since $(\beta+\gamma)>0$. Besides, in this case $\beta=\gamma=0.5$ and consequently the parameter $\gamma$ can be neglected in the Bouc-Wen model leading to a simpler identification procedure.

Finally, a brief reference regarding the rest of the Bouc-Wen parameters $\left(c_{0}, k_{0}\right.$ and $\left.\alpha\right)$ will be made. These parameters are related with the three physical components of the model and they essentially affect the height of the global hysteretic loop and the slope and width of the hysteresis "tails", i.e., the shape of the extension branch of the hysteretic curve.

Essentially, the parameter $\alpha$ is a scale factor and the parameters $c_{0}$ and $k_{0}$ are related with the representation of a conventional damper behavior. Figure 13 shows the influence of the parameter $\alpha$ in the global hysteretic loop (since this parameter is a multiplier of the evolutionary variable $z$ ). In this case, the parameter $\alpha$ is increased from $10 \mathrm{~N}$ to $60 \mathrm{~N}$ with increments of $10 \mathrm{~N}$ while the other parameters are kept constant $\left(A=20.0, \beta=\gamma=5.0, n=2.0\right.$ and $\left.c_{0}=k_{0}=0.0\right)$.

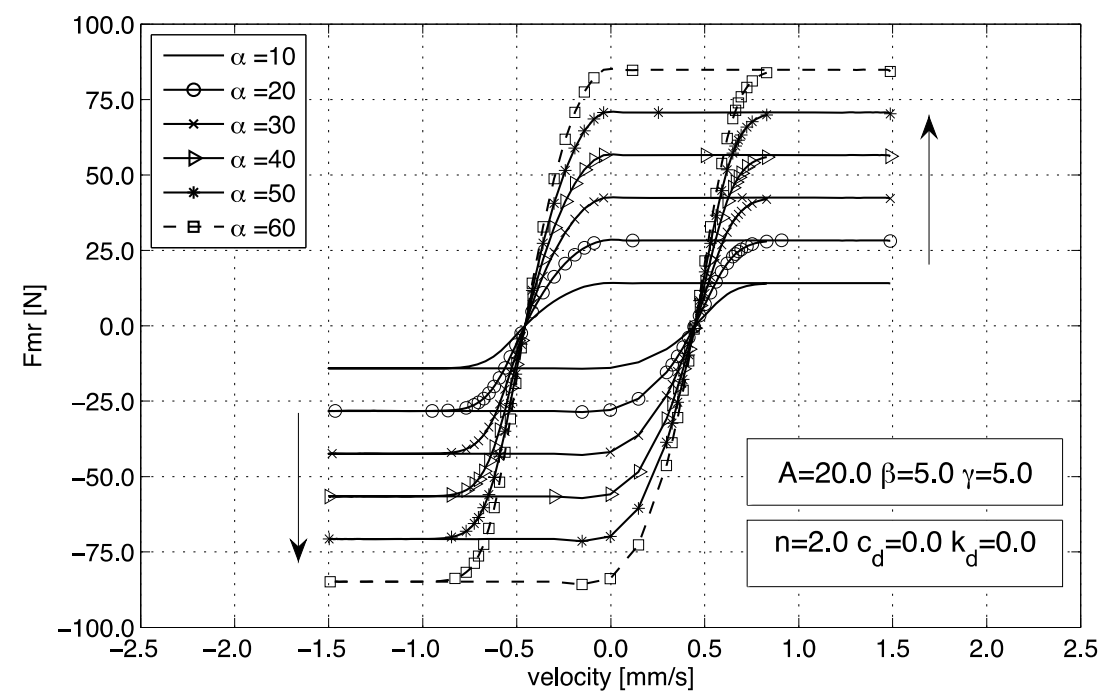

Figure 13: Influence of parameter $\alpha$ on force-velocity curves.

According with the resulting plot it is possible to verify that increasing the value of $\alpha$ will increase the height of the hysteresis loop. Thus, this parameter is responsible for the amplification of the vertical scale of the Bouc-Wen curve and can be seen as a scale factor of the hysteresis loop.

The parameter $c_{0}$ is a damping constant in the Bouc-Wen model that represents the postyield relationship between the damper force and the velocity and generates an inclined line with a slope defined by the parameter value. This parameter essentially controls the slope of the hysteresis loop external branches. The effect of $c_{0}$ in the hysteresis loop is shown in Figure 14 for constant values of $A=20.0, \beta=\gamma=5.0, n=2.0, \alpha=30$ and $k_{0}=0.0$. Changing the parameter causes a variation of the hysteresis loop slope with particular incidence in the external branch of the loop. Then, increasing the damping coefficient causes a rising in slope of the hysteresis tails and large values of $c_{0}$ provides a steep inclination. Finally, the effect of the spring parameter $k_{0}$ in the hysteresis loop is studied. 


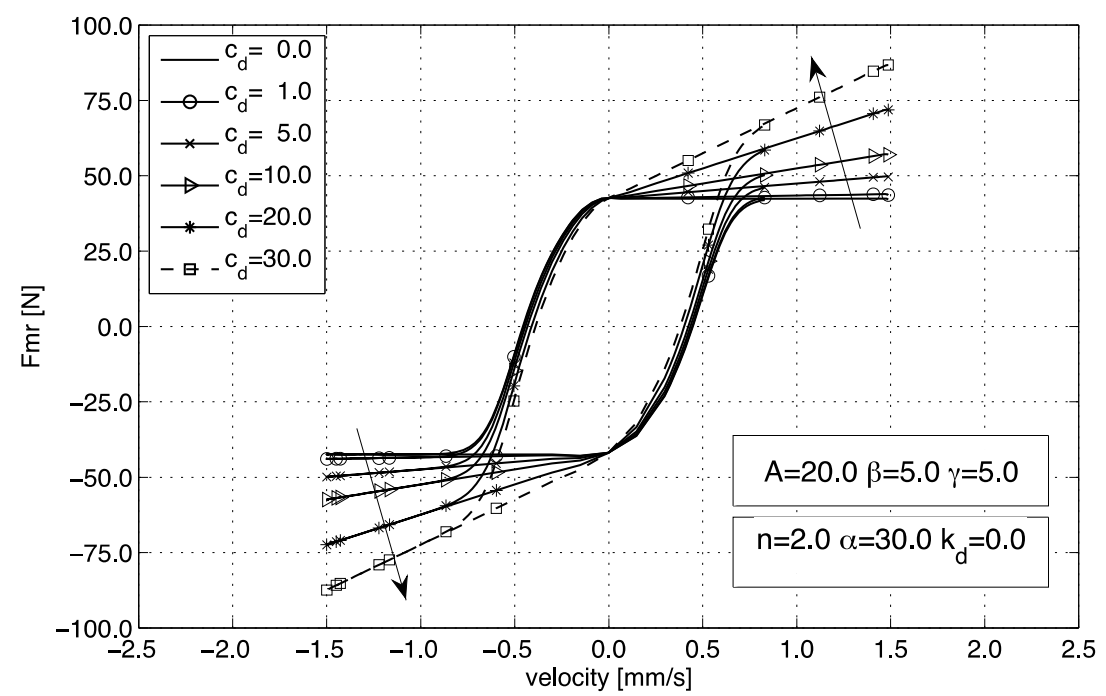

Figure 14: Influence of parameter $\mathrm{c}_{0}$ on force-velocity curves.

This parameter represents a stiffness parameter that generates a horizontal ellipse and is responsible for widening the ellipse shape around zero velocity area.

Figure 15 represents the influence of this parameter in the global hysteresis shape when $A=$ 20.0, $\beta=\gamma=5.0, n=2.0, \alpha=30$ and $c_{0}=10.0$. When the coefficient $k_{0}$ is increased, the spring component of the Bouc-Wen model will become more significant producing an enlargement of the area enclosed in the hysteresis loop. Large values of $k_{0}$ will produce a significant widening effect on the post-yield hysteresis ends instead of the pre-yield region.

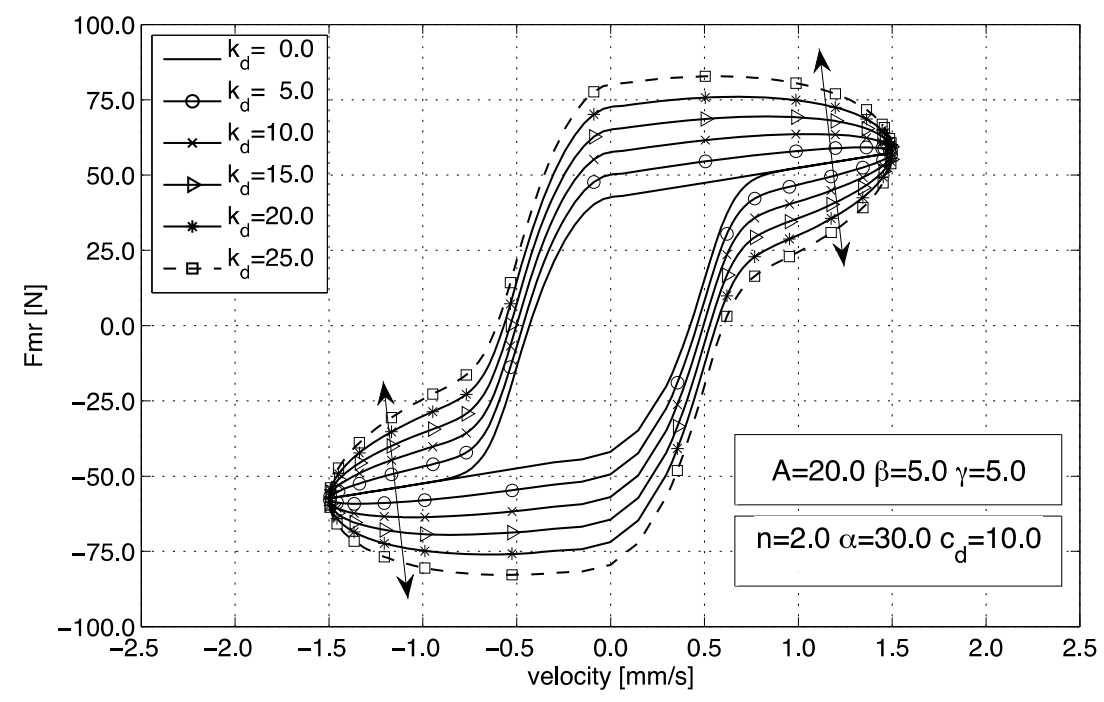

Figure 15: Influence of parameter $k_{0}$ on force-velocity curves.

The Modified Bouc-Wen model or Spencer model is a variation of the simple Bouc-Wen model presented previously. This model was developed to improve the nonlinear forcevelocity hysteretic response of MR dampers since the simple Bouc-Wen model does not reflect the roll-off effect in the region where the acceleration and velocity have opposite signs and the magnitude of the velocities are small [2]. 
This model includes a simple Bouc-Wen block and two new mechanical components: a spring and a dashpot connected as shown in Figure 3. The spring represents the accumulator stiffness and is characterized by the model parameter $k_{l}$; while the dashpot, defined by the model parameter $c_{1}$, is included in the model to create the roll-off effect that is observed in the experimental data at low velocities.

The effect of the model parameter $c_{1}$ in the hysteretic loops is displayed in Figure 16. The numerical responses were made for a variable value of $c_{l}$ while the rest of the model parameters are kept with a constant value. This analysis shows that increasing the value of $c_{0}$ produces an increase in the hysteretic loop slope along with an increase in the hysteretic loop width. Moreover, the characteristic " $\mathrm{S}$ " shape of the hysteretic loop is visible for moderate or high values of $c_{1}$ (for the present constant model parameters). In this case, the typical hysteretic loop is achieved when the value of $\mathrm{c} 1$ is significantly higher than the value of $c_{0}\left(c_{1}>10 c_{0}\right)$.

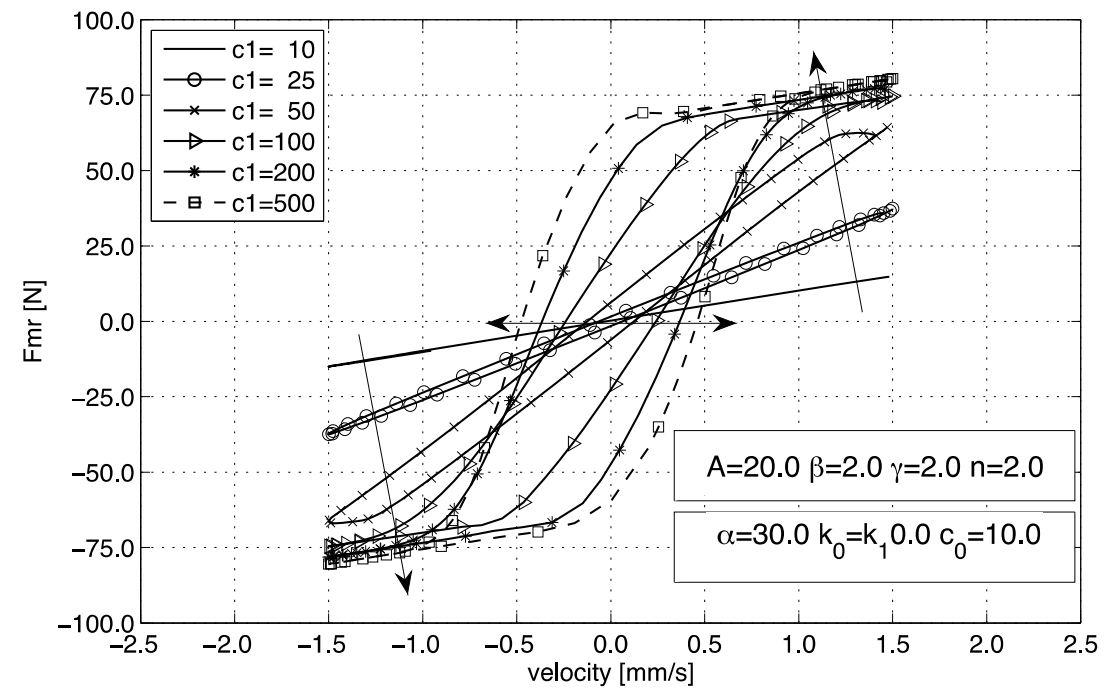

Figure 16: Influence of parameter $c_{1}$ on force-velocity curves

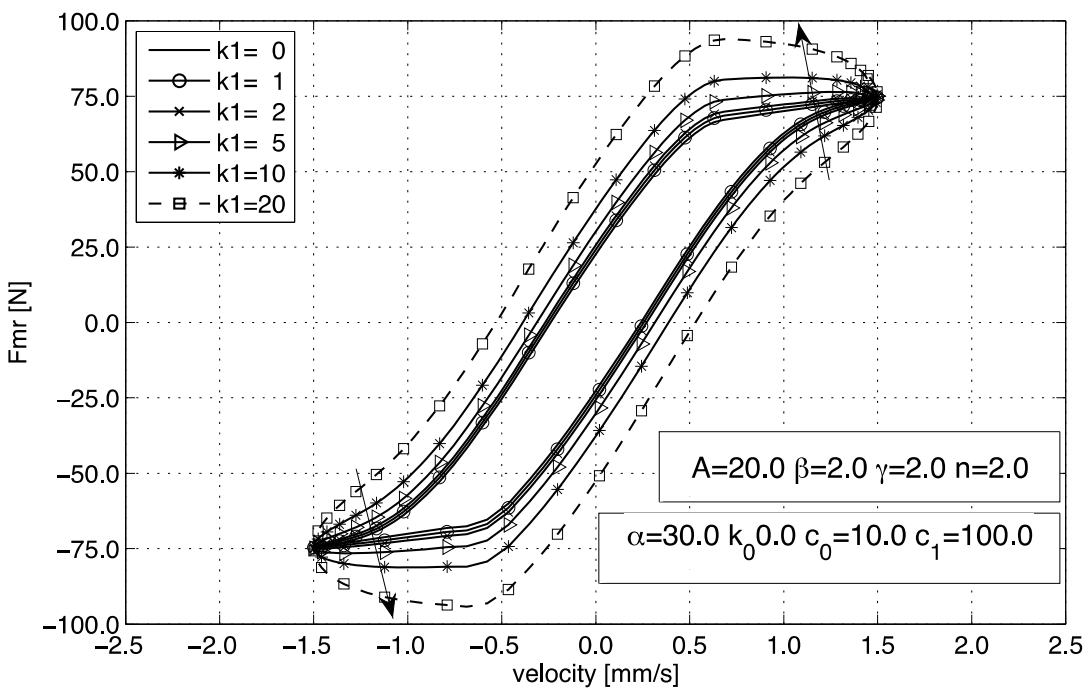

Figure 17: Influence of parameter $k_{l}$ on force-velocity curves 
Although the overall effect of this parameter can be characterized by the curves plotted in Figure 16, is important to notice that the effect of this parameter is also dependent on the rest of the Bouc-Wen model parameters and different shapes can be obtained by changing the other parameters. The roll-off effect observed in the experimental data at low velocities can be adjusted varying this parameter in combination with the Bouc-Wen parameters.

Finally, the effect of the spring parameter $k_{l}$ is shown in Figure 17. This parameter has a similar effect as $k_{0}$, i.e., increasing the value of $k_{l}$ produces a widening of the hysteretic loop.

The experimental results obtained with these MR damper show that this widening effect is insignificant, which suggests that the contribution of the linear springs to the shape of the hysteretic loop is negligible compared to other model components.

\section{PARAMETERS IDENTIFICATION}

High-accuracy models usually comprise a large number of parameters that require a reliable parameter identification procedure to efficiently estimate the suitable model parameters and reduce large parameter identification errors.

The estimation of the model parameters can be established based on several numerical approaches such as least square methods, fuzzy methods, neural networks, genetic algorithm, etc. The least square method is a simple and standard approach to reduce the difference between an experimental value and the fitted value provided by a dynamic model, and the application of this method is mostly appropriate for models that have linear variations in the parameters.

However, the dynamic models for MR dampers are nonlinear in the parameters and a nonlinear approach should be considered to estimate model parameters. Nonlinear least squares regression generalizes and extends the linear least squares regression model to a much larger and more general class of functions, especially functions that cannot be linearized and is often used to identify the non-linear parameters of the numerical models of MR dampers. Therefore, the non-linear least square regression model will be used in the present study to estimate the model parameters for several numerical models.

There are several computational routines to solve nonlinear curve-fitting problems in the MATLAB Optimization Toolbox [19]. After defining the identification procedure it is necessary to select the main numerical models that will be considered to simulate the MR damper response. In this case, the Modified Bouc-Wen model will be used for the RD-1005-3 MR damper. The force generated by the MR damper is defined by the seven parameters of the Bouc-Wen block $\left(A, \beta, \gamma, n, c_{0}, k_{0}\right.$ and $\left.{ }_{\alpha}\right)$ and two new model parameters $k_{l}$ and $c_{l}$ related with the new mechanical components. Thus, the identification procedure involves nine model parameters $\left(A, \beta, \gamma, \alpha, c_{0}, k_{0}, k_{1}, c_{1}\right.$, and $\left.n\right)$, or ten if the force offset $f_{0}$ (or $\left.x_{0}\right)$ is considered as a variable, to be identified and the parameter vector is then defined as

$$
\Theta=\left[\alpha, \beta, A, n, k_{0}, k_{1}, c_{0}, c_{1}, x_{0}\right]
$$

The damper has a softening hysteretic effect with no signs of hardening (case I, II or III, i.e., $(\beta+\gamma)>0)$ and the parameters $A, \alpha, k_{0}$ and $c_{0}$ are positive variables. The parameter $c_{0}$ should have a reduced value to avoid steep slopes and $k_{0}$ should be close to zero to reduce the widening effect. It was assumed that $n=2$ and the force offset, defined by $k_{l}\left(\mathrm{x}-x_{0}\right)$ that represent the force due to the accumulator existence, has the same value of the Bingham and the simple Bouc-Wen model force offset $\left(f_{0}=40 \mathrm{~N}\right)$. The parameter $c_{1}$ is much higher than $c_{0}(\mathrm{a}$ constrain $c_{1}>5 c_{0}$ was imposed in this study) and the spring parameter $k_{l}$ has a small value due to the reduced widening effect observed in the experimental data. The different hysteretic loops of the numerical model were determined by a set of model parameters for each experimental excitation case. 
Figure 18 shows the result that was obtained with the parameter identification procedure for the Modified Bouc-Wen model $(1.50 \mathrm{~Hz}, 4 \mathrm{~mm}$ and $0.75 \mathrm{~A})$.

This procedure was repeated for each set of experimental data and the values of the model parameters of the Modified Bouc-Wen model were determined for each dataset.

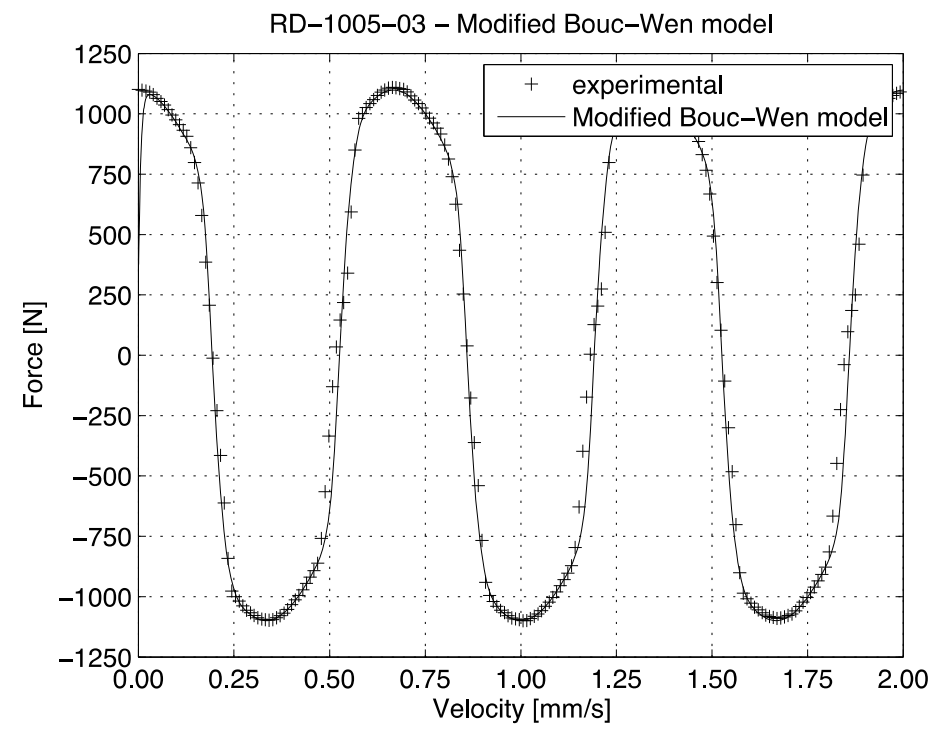

Figure 18: RD-1005-3 MR damper - Parameter identification of the Modified Bouc-Wen model $(1.50 \mathrm{~Hz}, 4 \mathrm{~mm}$ and $0.75 \mathrm{~A})$.

The average values of the current independent parameters of the Bouc-Wen model $A=10.013, \beta=3.044 \mathrm{~mm}^{-2}, \gamma=0.103 \mathrm{~mm}^{-2}$ and $k_{0}=1.121 \mathrm{~N} / \mathrm{mm}$ were found. The parameters $\alpha$, $c_{0}$ and $c_{1}$ are described as functions of the input current by a polynomial curve fitting procedure as shown in Figures 19-21.

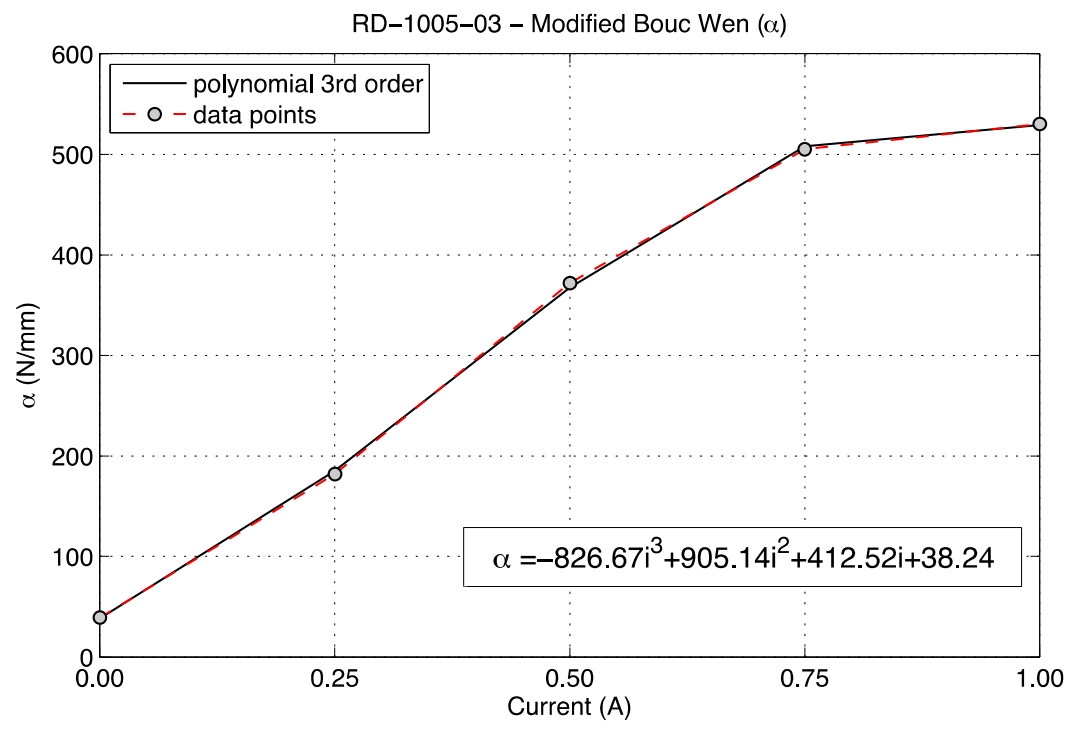

Figure 19: Curve fitting for $\alpha(I)$ of the Bouc-Wen model. 


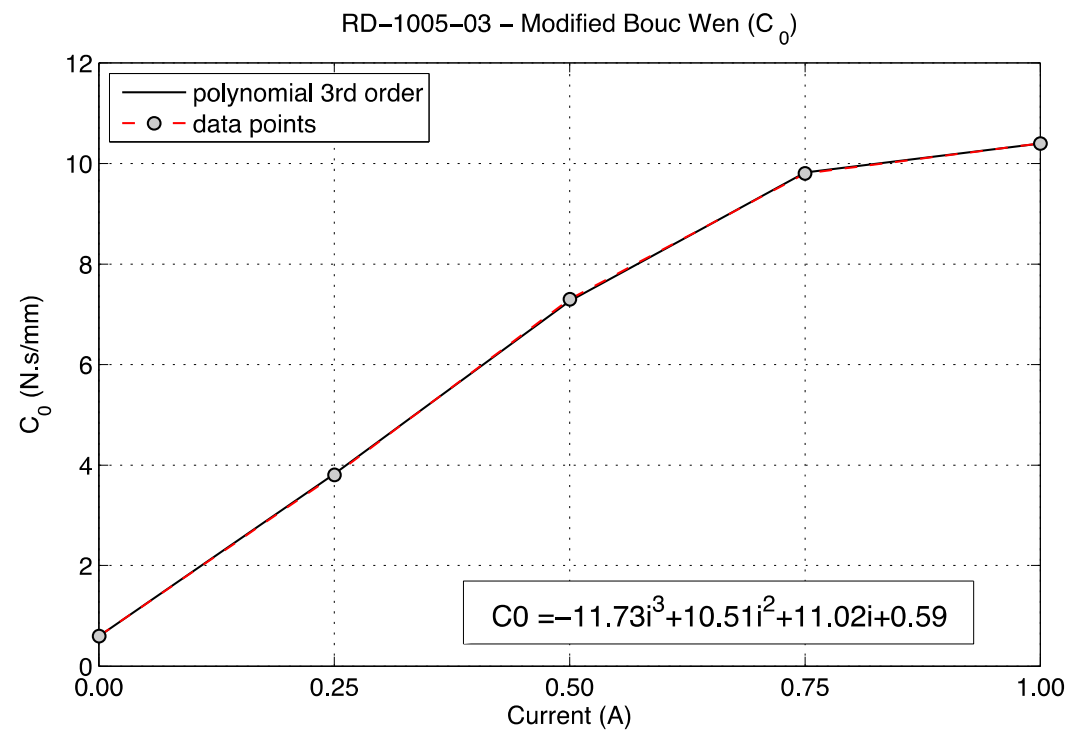

Figure 20: Curve fitting for $c_{0}(I)$ of the Bouc-Wen model.

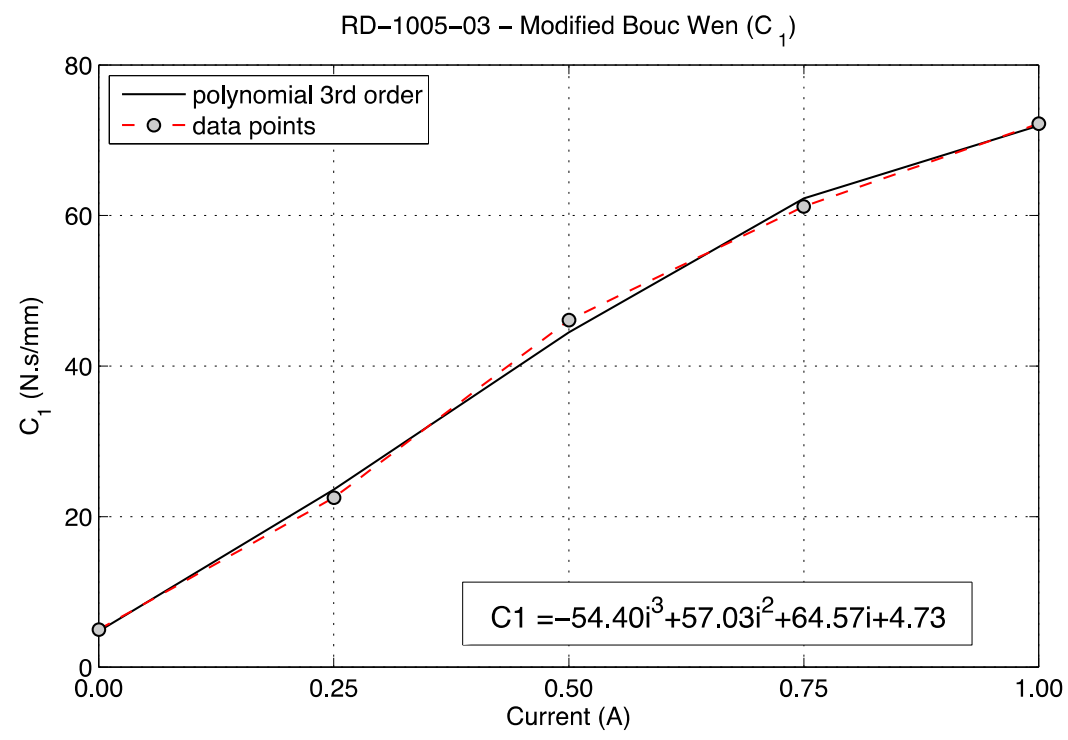

Figure 21: Curve fitting for $c_{I}(I)$ of the Bouc-Wen model.

According with the curve fitting procedure, the model parameters $\alpha, c_{0}$ and $c_{1}$ can be described by

$$
\begin{gathered}
\alpha(I)=-826.67 I^{3}+905.14 I^{2}+412.52 I+38.24 \\
c_{0}(I)=-11.73 I^{3}+10.51 I^{2}+11.02 I+0.59 \\
c_{1}(I)=-54.40 I^{3}+57.03 I^{2}+64.57 I+4.73
\end{gathered}
$$

Results of experimental and numerical responses of the MR damper are presented in Figure $22(1.5 \mathrm{~Hz}, 4 \mathrm{~mm}$ of amplitude and $I=1.00 \mathrm{~A})$. The results show that the Modified BoucWen model is capable to characterize the MR damper hysteretic response. 

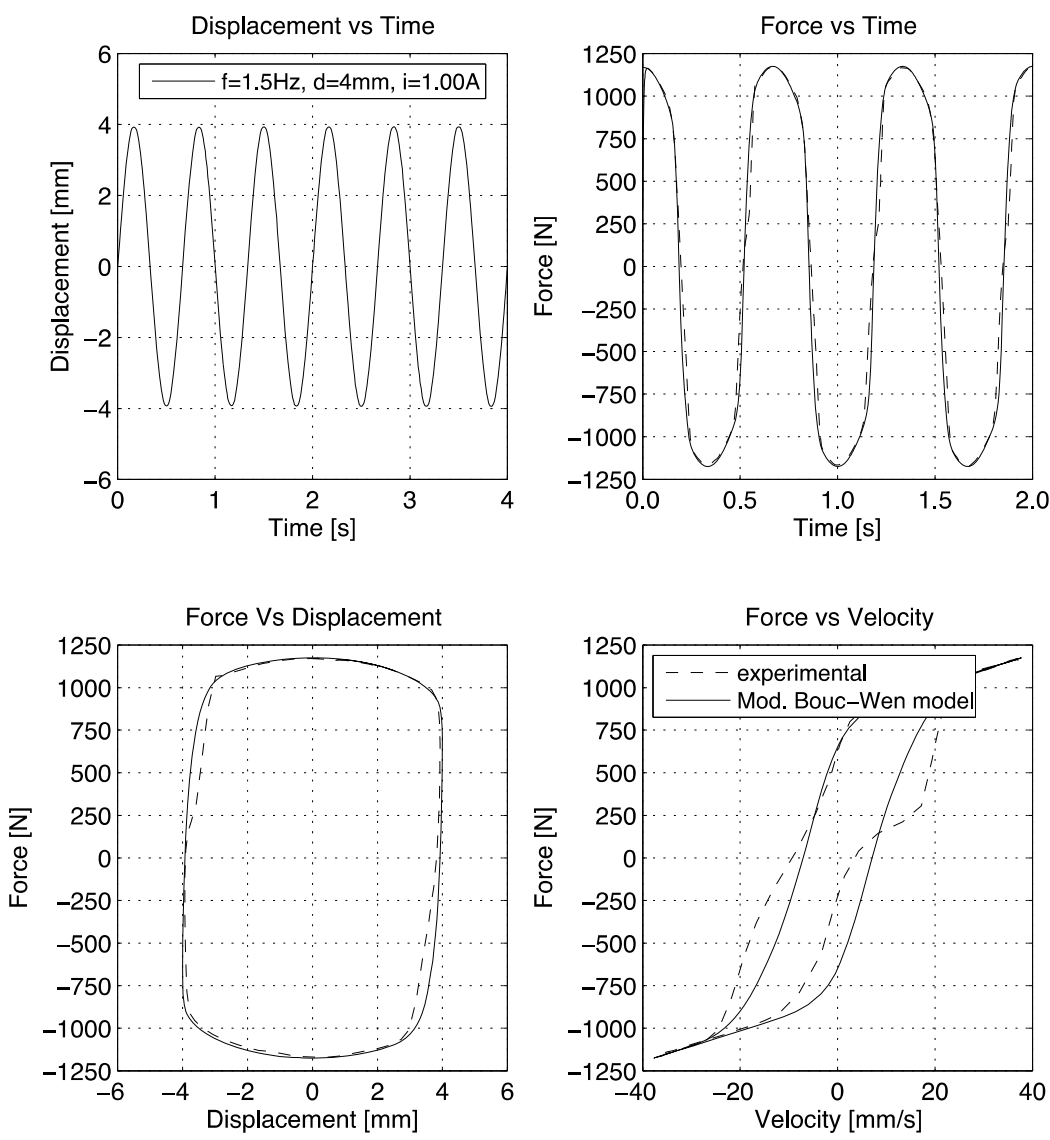

Figure 22: RD-1005-3 MR damper - Experimental vs. numerical response for the Bouc-Wen model

\section{CONCLUSIONS}

The present article addressed the non-linear hysteretic properties of MR dampers. An experimental testing procedure was carried out to characterize the response of a commercial MR damper and the experimental data were used to develop a numerical model. Some model parameters that must be initially found to construct a realistic numerical response. Thus, an identification routine was developed and the predicted response was compared with the experimental data. The results showed that the selected numerical model is capable to simulate the hysteretic behavior of the MR damper.

\section{REFERENCES}

[1] B. Sapiński, J. Filuś, Analysis of parametric models of MR linear damper, Journal of Theoretical and Applied Mechanics, 41-2, 215-240, 2003.

[2] J.M. Ginder, Rheology Controlled by Magnetic Fields. Encyclopedia of Applied Physics, 16, 487, 1996.

[3] M. Braz-Cesar, R. Barros, Semi-Active Control of an Experimental Frame using MR Dampers: numerical results and experimental validation, COMPDYN $2011-3^{\text {rd }}$ International Conference on Computational Methods in Structural Dynamics \& Earthquake Engineering, 2011. 
[4] M. Cesar, R. Barros, Semi-Active Vibration Control of a 3-DOF Scaled Frame with a Magneto-Rheological Damper, CST2010 - The Tenth International Conference on Computational Structures Technology, 2010.

[5] M. Braz-César, R. Barros, Semi-active Vibration Control of Buildings using MR Dampers: Numerical and Experimental Verification, $14^{\text {th }}$ ECEE $-14^{\text {th }}$ European Conference on Earthquake Engineering, 2010.

[6] M. Braz-César, R. Barros, Properties and Numerical Modeling of MR Dampers, $15^{\text {th }}$ ICEM - $15^{\text {th }}$ International Conference on Experimental Mechanics, Porto, 2012.

[7] D.H. Wang, W.H. Liao, Magnetorheological fluid dampers: a review of parametric modelling, Smart Materials and Structures, 20, 023001, 2011.

[8] F. Gandhi, W. Bullough, On The Phenomenological Modeling of Electrorheological and Magnetorheological Fluid Preyield Behavior, Journal of Intelligent Material Systems and Structures, 16- 3, 237-248, 2005.

[9] F. Goncalves, J. Koo, M. Ahmadian, A Review of The State of the Art in Magnetorheological Fluid Technologies - Part I: MR Fluid and MR Fluid Models, The Shock and Vibration Digest, 38- 3, 203-219, 2006.

[10] S. Guo, S. Yang, C. Pan, Dynamic Modeling of Magnetorheological Damper Behaviors, JIMSS, 17-1, 3-14, 2006.

[11] M. Maślanka, B. Sapiński, Experimental Study of Vibration Control of a Cable with an Attached MR Damper, Journal of Theoretical and Applied Mechanics, 45-4, 893-917, 2007.

[12] B.F. Spencer Jr., S.J. Dyke, M.K. Sain, J.D. Carlson, Phenomenological model of a magnetorheological damper, Journal of Engineering Mechanics, 123, 230-238, 1997.

[13] M.T. Avraam, MR-fluid brake design and its application to a portable muscular rehabilitation device, $\mathrm{PhD}$ thesis, 2009.

[14] M. Kamath, H. Hurt, N. Wereley, Analysis and Testing of Bingham Plastic Behavior in Semi-Active Electrorheological Fluid Dampers, Smart Material \& Structures, 5, 576590, 1996.

[15] Y.K. Wen, Method for random vibration of hysteretic systems, Journal of the Engineering Mechanics Division, 102-2, 249-263, 1976.

[16] W. Schwanen, Modelling and identification of the dynamic behavior of a wire rope spring, Masters thesis, 2004.

[17] C.W. Wong, Y.Q. Ni, J.M. Ko, Steady-State oscilliation of hystereric differential model. I: Response analysis, Journal of engineering mechanics, 120, 2271-2298, 1994.

[18] F. Ikhouane, J. Rodellar, Systems with hysteresis: Analysis, identification and control using the Bouc-Wen model, John Wiley \& Sons, 2007.

[19] The Mathworks, Inc. Optimization Toolbox User's Guide, USA, 2012. 